\title{
Non-invasive transdermal delivery of chemotherapeutic molecules in vivo using superparamagnetic iron oxide nanoparticles
}

\author{
Vanisri Raviraj ${ }^{1,2 \dagger}$, Binh T. T. Pham ${ }^{1,5}$, Byung J. Kim, Nguyen T. H. Pham¹', Lai F. Kok ${ }^{3}$, Nicole Painter ${ }^{1}$, \\ Naomi C. Delic ${ }^{2}$, Stephen K. Jones ${ }^{4}$, Brian S. Hawkett ${ }^{1+}$ and J. Guy Lyons 2,6, $^{7^{*+}}$ (D)
}

${ }^{*}$ Correspondence:

guy.lyons@sydney.edu.au

Vanisri Raviraj and Binh T.T.

Pham contributed equally

${ }^{\dagger}$ Brian S. Hawkett and J. Guy

Lyons are co-senior authors

${ }^{7}$ Immune Imaging,

Centenary Institute, The

University of Sydney, Royal

Prince Alfred Hospital, Bldg

93, Camperdown, NSW,

Australia

Full list of author information

is available at the end of the article

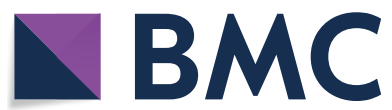
adaptation, distribution and reproduction in any medium or format, as long as you give appropriate credit to the original author(s) and the source, provide a link to the Creative Commons licence, and indicate if changes were made. The images or other third party material in this article are included in the article's Creative Commons licence, unless indicated otherwise in a credit line to the material. If material is not included in the article's Creative Commons licence and your intended use is not permitted by statutory regulation or exceeds the permitted use, you will need to obtain permission directly from the copyright holder. To view a copy of this licence, visit http://creativecommons.org/ licenses/by/4.0/. The Creative Commons Public Domain Dedication waiver (http://creativecommons.org/publicdomain/zero/1.0/) applies to the data made available in this article, unless otherwise stated in a credit line to the data. 
in the liver (Prausnitz and Langer 2008; Alkilani et al. 2015; Palmer and DeLouise 2016). In the case of skin diseases, transdermal delivery can enable local concentrations of drugs to achieve therapeutic levels while keeping the systemic levels very low, thereby reducing off-target toxicity. However, delivery of therapeutic molecules through the skin poses particular challenges. In particular, the stratum corneum (SC) is impermeable to almost all compounds larger than $500 \mathrm{Da}$ (Matsui and Amagai 2015).

The past two decades have seen the development of techniques to improve the penetration of drugs through the skin, including iontophoresis (Cazares-Delgadillo et al. 2007), sonophoresis (Alvarez-Roman et al. 2003), electroporation (Lombry et al. 2000), microneedles (Prausnitz 2004), magnetophoresis (Murthy et al. 2010), microdermabrasion (Herndon et al. 2004) and electron beam irradiation (Kotiyan et al. 2004). However, the undesirable side effects of these techniques include skin irritation, pain and potential for infection.

Nanoparticles have the potential to significantly improve the penetration of large molecules across the SC, thereby improving bioavailability without compromising the skin barrier to pathogenic microbes. Nanoparticles can improve penetration of drugs by acting either as carriers, in which the hydrophobicity or stability characteristics of a pre-formed nanoparticle-drug complex is improved relative to the drug alone, or as co-administered adjuvants, which change the behavior of the tissues to enhance their penetrability. They can offer stability, biodegradability, low toxicity, and biocompatibility (Dianzani et al. 2014; Wang et al. 2016; Pelaz et al. 2017; Carter et al. 2019). However, their size can be an impediment to transdermal applications. Generally, larger nanoparticles $(20-30 \mathrm{~nm})$ can penetrate only through hair follicles (Zvyagin et al. 2008), limiting their usefulness for transdermal applications. In contrast, nanoparticles smaller than $10 \mathrm{~nm}$ can permeate through both the SC and hair follicles (Hansen and Lehr 2012). However, aggregation of small nanoparticles in vivo can limit their tissue penetration and increase toxicity (Pelaz et al. 2017). The surface charge of nanoparticles also affects their ability to penetrate the skin.

Melanoma is the most aggressive common skin cancer with a 5-year survival rate of $10 \%$ among metastatic melanoma patients (Garbe et al. 2011; Mundra et al. 2015). In recent years, immunotherapies and chemotherapies targeted to specific mutant proteins have revolutionized the therapy of melanoma, but lack of response and relapse through resistance are common and so conventional cytotoxic chemotherapies still play a major role in its management (Tang et al. 2017; Vanella et al. 2019). Several chemotherapeutic drugs are available for melanoma treatment, including dacarbazine, temozolomide, 5-fluorouracil (5-FU), paclitaxel and platinum compounds. However, the overall response rate is less than $20 \%$ and each of these agents produces unwanted systemic side effects. Applications of nanoparticles to the treatment of melanoma is of growing interest (Tang et al. 2017), despite the aforementioned drawbacks of size, charge and aggregation inherent in many nanoparticles. Carbon nanotubes loaded with doxorubicin (DOX) induced melanoma cell death in vitro and abrogated tumor growth in xenograft mouse models (Chaudhuri et al. 2010). Inorganic nanoparticles based on silica and aluminum (Oh et al. 2012; Yu et al. 2013), protein-based nanoparticles (Vannucci et al. 2012) and DOX-loaded liposomes (Lohade et al. 2016) have also been investigated for melanoma treatment. 
Superparamagnetic iron oxide nanoparticles (SPIONs) have been successfully used in various biomedical applications that benefit from their magnetic properties, biocompatibility, low toxicity, easy separation methodology and flexibility of surface modification (Shubayev et al. 2009; Veiseh et al. 2010; Kievit et al. 2011; Hasany et al. 2012; Ali et al. 2016; Palanisamy and Wang 2019). However, to date, no SPIONs have been commercialized successfully for drug delivery purposes. We have developed a unique coating technology for SPIONs that produces a highly stable dispersion for biomedical applications (Jain et al. 2010; Eamegdool et al. 2014; Pham et al. 2015, 2017). These SPIONs are nontoxic in vitro and in vivo and can be readily tailored to have different coating compositions and functional groups at the end of stabilizers (Bryce et al. 2013; Eamegdool et al. 2014; Pham et al. 2018). We have shown that co-administration of our nanoparticles with a cytotoxic drug can enhance the penetration of the drug in an in vitro spheroid model (Bryce et al. 2013).

In this study, we investigated the potential of our sterically stabilized SPIONs as chemotherapy adjuvants in transdermal drug delivery in vivo. We found that topical co-administration of these SPIONs improved transdermal delivery and the anti-tumor responses of mouse melanomas to chemotherapeutic drugs.

\section{Methods}

\section{Reagents}

Reversible addition-fragmentation chain-transfer (RAFT) agents 2-[(butylsulfanyl)carbonothioyl]sulfanyl propanoic acid $\left(\mathrm{C}_{4}\right.$-RAFT) and methoxy-polyethylene glycol modified 2-[(butylsulfanyl)carbonothioyl]sulfanyl propanoic acid were kindly provided by $\mathrm{Dr}$ Algi Serelis (DuluxGroup). Fe(II) chloride tetrahydrate (99\%), Fe(III) chloride hexahydrate (98\%), Fe(III) nitrate nonahydrate (99\%), acrylamide ( $>98 \%)$, rhodamine B isothiocyanate $(>70 \%)$, DOX $(>98 \%)$ and nitric acid $\left(\mathrm{HNO}_{3}, 65 \%\right.$, Suprapur ${ }^{\circledR}$, Millipore) were purchased from Sigma-Aldrich (St. Louis, USA). Ammonium hydroxide $\left(28 \% \mathrm{NH}_{3}\right.$ in water) and sodium hydroxide $(\mathrm{NaOH})(>98 \%)$ were obtained from Ajax Finechem (Australia). 1,4-Dioxane (Fluka-Sigma-Aldrich, St Louis, USA), monoacryloxyethyl phosphate (MAEP, >98\%, PolySciences, Warrington, USA) and 4,4-azobis(4-cyanovaleric acid) (Wako, USA) were used as received.

\section{Nanoparticles}

Maghemite nanoparticles $\left(\gamma-\mathrm{Fe}_{2} \mathrm{O}_{3}\right.$, average $25 \mathrm{~nm}$ core diameter, unless stated otherwise) were synthesized by the coprecipitation method (Massart et al. 1995) and sterically stabilized with a mixture of $90 \%$ poly(ethylene glycol) methyl ether (MPEG)-end and $10 \% \mathrm{NH}_{2}$-end macro-RAFT copolymers, prepared using RAFT polymerization (Pham et al. 2018). The polymers each contain a block of 10 units of MAEP to anchor MPEG or polyacrylamide to the SPIONs' surface, and either a block of 40 units of acrylamide and 17 units of poly(ethylene glycol) (PEG) (in MPEG-end-copolymer) or a block of 60 units of acrylamide (in $\mathrm{NH}_{2}$-end copolymer) for steric stabilization. We refer to these copolymer-coated nanoparticles as " $10 \% \mathrm{NH}_{2}$-SPIONs". Transmission electron microscopy images were obtained using a JEOL 1400 microscope. Hydrodynamic diameter was measured by dynamic light scattering (DLS) using Malvern's Zetasizer nano-ZS. The polymer content was $13 \%(\mathrm{w} / \mathrm{w})$ of the dried solid as analyzed by thermogravimetric 
analysis (Additional file 1: Figure S1d). Iron concentration of the SPIONs was determined by flame atomic absorption spectroscopy (Varian AA800 spectrometer). For in vivo studies, $10 \% \mathrm{NH}_{2}$-SPIONs were dispersed in PBS to the final concentration of $5 \mathrm{mg} / \mathrm{mL}$ as Fe and then filtered through a $0.22 \mu \mathrm{m}$ sterile syringe filter.

Rho-SPIONs were made by labeling $10 \% \mathrm{NH}_{2}$-SPIONs with rhodamine $\mathrm{B}$ isothiocyanate in PBS at room temperature in the dark for $4 \mathrm{~h}$. Free rhodamine was removed using Amicon Ultra MWCO 3000 centrifugation filter units. Fluorescence intensity of Rho-SPIONs was measured with a RF-5301 PC spectrofluorophotometer (Shimadzu, Kyoto, Japan).

\section{Cell culture}

Amelanotic tyrosinase-knockout B16-F10 melanoma cells were kindly provided by Dr Shweta Tikoo from the Centenary Institute, Sydney, Australia, and maintained in Advanced Dulbecco's modified Eagle's medium and 5\% fetal bovine serum (Thermo Fisher Scientific, Waltham, USA). They were stably transfected with CEFLP-tdTomato-H2B eukaryotic expression plasmids using GenePORTER ${ }^{\circledR} 3000$ (Genlantis, San Diego, CA, USA). Transfected cells were selected with puromycin $(10 \mu \mathrm{g} / \mathrm{mL})$ and tdTomato-expressing cells were purified by flow cytometry on a BD Influx Cell Sorter.

\section{Animals}

A colony of hairless albino C57BL/6 mice was established from a breeding pair obtained from the Jackson Laboratory (strain 017840) and maintained by breeding homozygous males $(\mathrm{Hr}-/-)$ with heterozygous $(\mathrm{Hr} \pm)$ females. Mice were housed with dust-free bedding (Able Scientific, Australia), regular chow, water, environmental enrichment and 12/12 h light/dark cycles.

\section{Transdermal delivery of SPIONs and chemotherapeutic drugs}

Mice were anesthetized by intraperitoneal injection of ketamine $(75 \mathrm{mg} / \mathrm{kg})$ and medetomidine $(1 \mathrm{mg} / \mathrm{kg})$, placed on a warming pad and the back skin of the mice was cleaned with a $70 \%$ isopropyl alcohol swab (Livingstone International, Australia). Rho-SPIONs ( $20 \mu \mathrm{l}$ of $2 \mathrm{mg} / \mathrm{mL}$ in PBS) were applied to the skin and left for $1 \mathrm{~h}$, after which excess liquid was wiped off with a wet cotton bud. PBS only $(20 \mu \mathrm{L})$ was used for the control mouse. For chemotherapeutic drug delivery, a $30 \mu \mathrm{L}$ mixture in PBS containing either both $10 \% \mathrm{NH}_{2}$-SPIONs $(1.3 \mathrm{mg} / \mathrm{mL}$ as Fe) and DOX $(0.17 \mathrm{mM}), 10 \%$ $\mathrm{NH}_{2}$-SPIONs only $(1.3 \mathrm{mg} / \mathrm{mL}$ as $\mathrm{Fe})$, or DOX only $(0.17 \mathrm{mM})$, was applied to the mouse skin and then left for $1-2 \mathrm{~h}$, at which time unabsorbed material was removed by wiping. Anesthesia was reversed with atipamezole $(0.2 \mathrm{mg} / \mathrm{kg})$.

The mice were imaged under anesthesia on a Carestream In Vivo FX PRO (Carestream Health, Woodbridge, USA) using $550 \mathrm{~nm}$ excitation and $600 \mathrm{~nm}$ emission wavelength filters before application and 1, 2, 24 and $48 \mathrm{~h}$ after application. They were then euthanized, and treated skins were fixed in $4 \%$ paraformaldehyde (PFA) overnight at $4{ }^{\circ} \mathrm{C}$ for fluorescent imaging. 


\section{In vivo melanoma model}

Eight-week-old hairless albino C57BL/6 mice were injected subcutaneously with $1 \times 10^{4}$ B16F10-tdTomato amelanotic melanoma cells. Three days post-injection, the mice were randomized into different treatment groups. Each group was topically treated at the tumor site 3 times/week with $60 \mu \mathrm{L}$ of either vehicle $(0.9 \%$ saline), 5 -FU (1 mM) alone, $10 \% \mathrm{NH}_{2}$-SPIONs $(1 \mathrm{mg} / \mathrm{mL})$ alone, or a mixture of 5 -FU $(1 \mathrm{mM})$ and $10 \% \mathrm{NH}_{2}$-SPIONs $(1 \mathrm{mg} / \mathrm{mL})$. Tumor growth was measured using calipers and micrographs taken with a Dino-Lite AM4515ZT Edge Handheld Microscope. Tumor volume was calculated by the modified ellipsoidal formula (Tomayko and Reynolds 1989). Mice were euthanized when tumors reached $1 \mathrm{~cm}^{3}$, or skin ulceration appeared to be imminent.

\section{Histology and imaging}

PFA-fixed skin samples from Rho-SPION-treated mice were embedded in Tissue-Tek ${ }^{\circledR}$ OCT (Sakura, Torrance, CA), frozen and cut into $7-\mu \mathrm{m}$-thick sections. Sections were counterstained with $1 \mu \mathrm{g} / \mathrm{mL}$ for $5 \mathrm{~min}$ 4',6-diamidino-2-phenylindole (DAPI) (SigmaAldrich, St Louis, MO, USA), washed 3 times with PBS, mounted in Prolong Gold ${ }^{\circledR}$ antifade mounting media (Life Technologies, Carlsbad, CA) and cover-slipped. Skin sections were stained with F4/80 primary antibody (1:200, SC-52664, BM8 clone, Santa Cruz Biotechnology, Dallas, USA) and goat anti-rat IgG $(\mathrm{H}+\mathrm{L})$ Alexa Fluor 488-for secondary Antibody (1:200, A-11006, ThermoFisher Scientific, Australia) to detect macrophage distribution in the skin. Images were acquired using an Olympus FV1000 confocal microscope (Olympus, Japan) and Leica SP5 and SP8 confocal microscopes (Leica Biosystem, Wetzlar, Germany).

B16-F10 melanoma tumors were fixed in 4\% PFA overnight, embedded in paraffin, and cut into 4- $\mu \mathrm{m}$-thick sections for hematoxylin and eosin (H\&E) staining. The sections were deparaffinized, rehydrated and then incubated in an antigen retrieval solution (RD913M, pH 6.0, Pacheco, USA) for 20 min over a boiling water bath to retrieve antigen. Endogenous peroxidase activity was blocked using $0.3 \%(\mathrm{w} / \mathrm{v})$ hydrogen peroxide. Sections were incubated for $1 \mathrm{~h}$ at room temperature with antibodies: 1:25 anti-F4/80 monoclonal rat antibody (SC-52664, Santa Cruz Biotechnology, Dallas, USA) or the rat IgG2a isotype control (559073, R35-95 clone, BD Biosciences, North Ryde, Australia) and 1:75 anti-CD31 monoclonal rabbit antibody (77699T, D8V9E clone, Cell Signaling Technology, Danvers, USA) or the rabbit IgG isotype control (ab172730, EPR25A clone, Abcam, Melbourne, Australia). Detection was with Rat-on-Mouse HRP-Polymer, Biocare Medical (Pacheco, USA) or anti-rabbit EnVision + system HRP-polymer, and diaminobenzidine as substrate (Dako-Agilent, Santa Clara, USA). Slides were counterstained with Mayer's haematoxylin.

\section{Image analysis}

Cell morphology of H\&E stained sections were characterized by the pathologist (LFK) to assess tumor borders and areas of tumor tissue necrosis. The pathologist was blinded to the treatments the specimens had received. Three mice per group were used for analysis and 4-6 regions were selected randomly in each tumor. Necrosis and whole tumor areas were measured using ImageJ software, and the percentage of the necrotic region was calculated by the total tumor area. Tumor-infiltrating lymphocytes were identified 
by a small cytoplasm:nucleus ratio, an oval, dark stained nucleus and a cell diameter of $7-10 \mu \mathrm{m}$ and were counted manually using ImageJ.

The vascular area was quantified from CD31-stained images in which the diaminobenzidine signal had been segmented by spectral deconvolution (Ruifrok and Johnston 2001) and thresholded using Fiji image analysis software. The image representing CD31 staining was then binarized and holes (vascular lumens) were filled. The CD31-positive stained area and total tumor area were measured in each tumor section, from which the percentage of CD31-stained area was calculated.

Statistical analyses were done using GraphPad Prism v. 8.4.

\section{Results}

\section{SPIONs penetrate mouse skin}

SPIONs were synthesized from maghemite particles by coating them with polyacrylamide and PEG copolymers, $10 \%$ of which were $\mathrm{NH}_{2}$-end derivatized for steric stabilization (Additional file 1: Figure S1a). The particles had a core diameter of 20-30 nm, a hydrodynamic diameter of $72 \mathrm{~nm}$, a polymer content of $13 \% \mathrm{w} / \mathrm{w}$, a polydispersity index of 0.11 and a zeta potential of $-40 \mathrm{mV}$ (Additional file 1: Figure S1b-e). To evaluate the penetration of SPIONs into mouse skin, fluorescent rhodamine-labeled SPIONs (RhoSPIONs) (Additional file 1: Figure S1f) were topically applied to hairless albino C57BL/6 mice. Live-mouse images (Fig. 1a, Additional file 1: Figures S2, S3) show a strong rhodamine fluorescent signal on the mouse's skin after one hour of application. After $24 \mathrm{~h}$, the signal intensity of the Rho-SPIONs was lower and dispersed over a larger area. No fluorescent signal above autofluorescence was detected on the control mouse. Fluorescence microscopy of fixed skin from the application areas of Rho-SPION-treated mice show that SPION particles penetrated through the SC within $24 \mathrm{~h}$ of application, accumulating most strongly in the epidermis, but also penetrating and being retained throughout the dermis (Fig. 1b, Additional file 1: Figure S3).

\section{SPIONs improve the transdermal penetration of doxorubicin}

To test whether topical application of SPIONs enhances the penetration of chemotherapy drugs into the skin, we topically applied the chemotherapeutic DNA-intercalating drug, doxorubicin (DOX, molecular mass 544), with and without SPIONs to the skin of the dorsal back and ear. The fluorescent properties of DOX enabled microscopic and macroscopic visualization of drug penetration into the mouse skin.

Live-mouse imaging $2 \mathrm{~h}$ after topical application showed strong DOX fluorescence in the application area for DOX applied with or without SPIONs, which decreased after 24 and $48 \mathrm{~h}$ (Fig. 2a). Macroscopically, the DOX signal was higher in the area treated with DOX alone compared with the area treated with both DOX and SPIONs at 2, 24 and $48 \mathrm{~h}$ after application. Confocal microscopy of cryosections of fixed tissue was used to determine the extent of penetration of DOX into the skin (Fig. 2b, Additional file 1: Figure S4). Mice treated with DOX alone showed poor penetration of DOX into the skin whereas mice treated with both DOX and SPIONs showed a strong DOX signal within the epidermis that extended into the dermis. It should be noted that DOX remaining on the surface of the epidermis would not have intercalated into cell DNA and therefore may have been lost during the fixation and counterstaining process; this would explain 

Time since application of Rho-SPIONs

Before

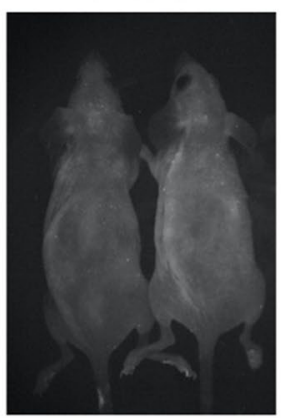

PBS

b
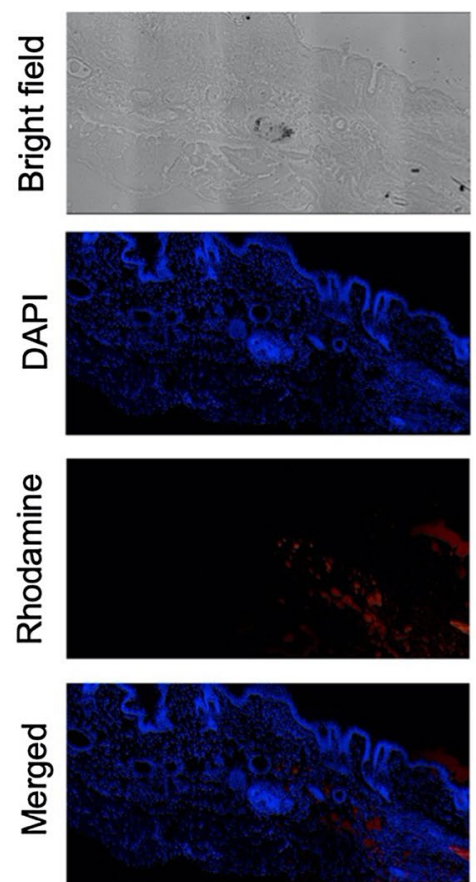

$1 \mathrm{~h}$ after

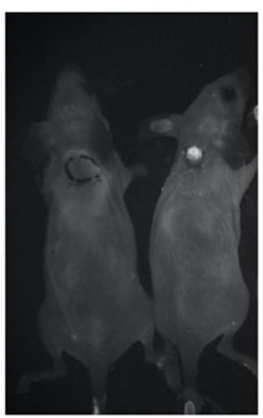

$24 \mathrm{~h}$ after

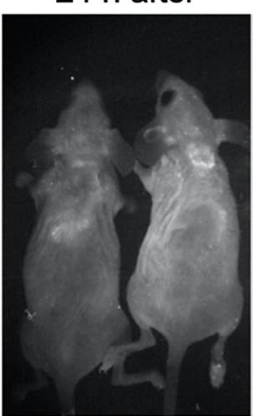

Rho-SPIONs
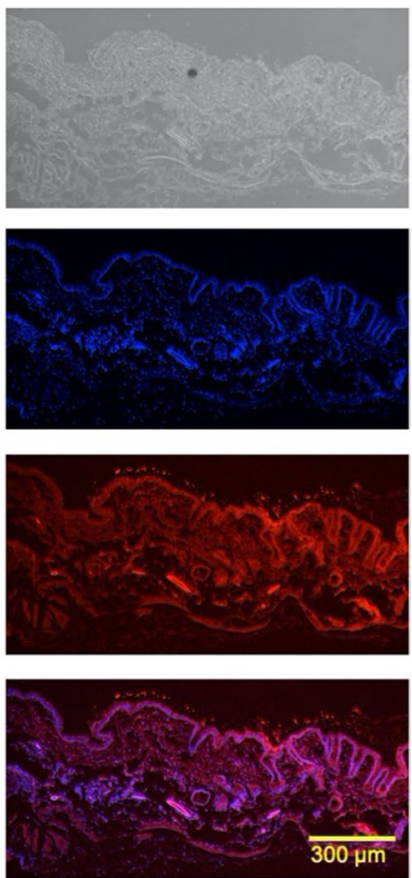

Fig. 1 Rhodamine-labeled SPIONs penetrate mouse skin. To examine the penetration of topically applied SPIONs into the skin, rhodamine-labeled SPIONs ( $2 \mathrm{mg} / \mathrm{mL}$ in $20 \mu \mathrm{L} \mathrm{PBS}$, mouse on the right) or vehicle ( $20 \mu \mathrm{L}$, mouse on the left) were applied to the back skin of mice. One hour after application, unabsorbed solution was removed by wiping. a Mouse whole-body fluorescence images were acquired the time points indicated. $\mathbf{b}$ Fluorescence micrographs of frozen fixed sections of the Rho-SPION-treated area of skin, $24 \mathrm{~h}$ after application, showing brightfield, nuclear counterstain (DAPI) and rhodamine signals. Representative images of 1-2 mice and experiment repeated 3 times

why there is no DOX signal detected in the DOX + PBS cryosections despite a strong signal being observed at the application site in the whole animal images.

\section{Co-application with SPIONs improves the response of melanoma tumors to 5-FU}

5-FU is a hydrophilic, negatively charged molecule that cannot easily penetrate through the hydrophobic, negatively charged SC (Fang et al. 2004). To determine whether SPIONs could improve the response to transdermal treatment by 5 -FU, we 
a Time since application of DOX and/or SPIONs

$2 \mathrm{~h}$

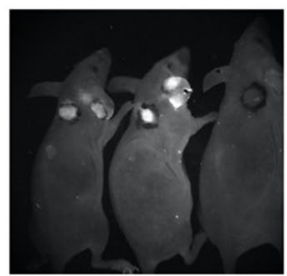

b
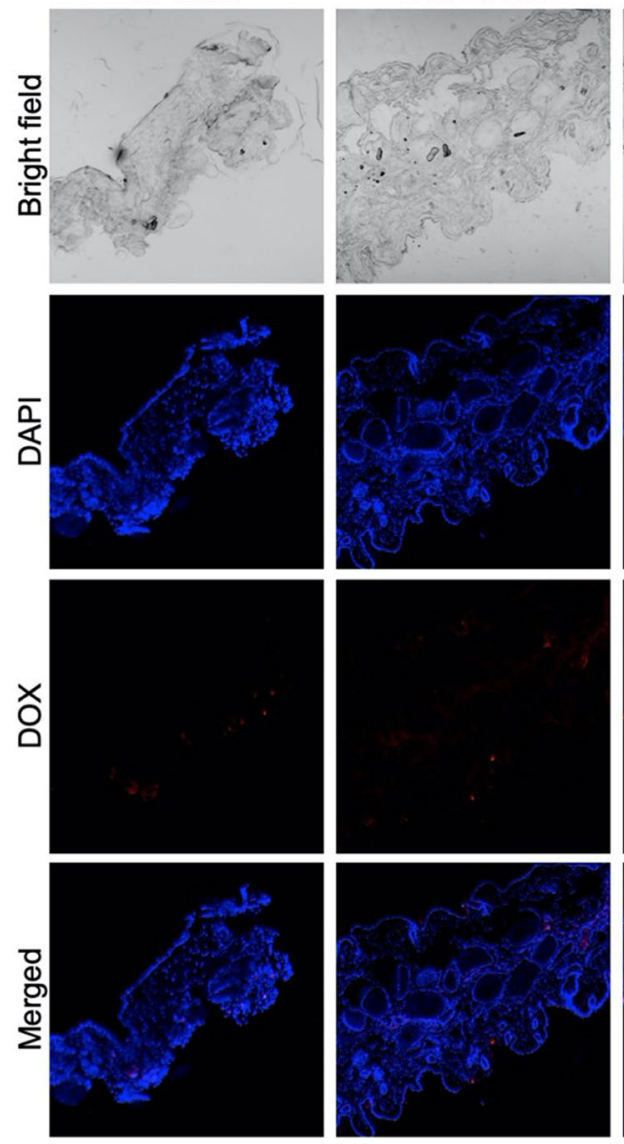

Fig. 2 SPIONs improve the penetration of DOX into mouse skin. a Unlabeled SPIONs alone (mouse on right), DOX alone (mouse in middle) or unlabeled SPIONs plus DOX (mouse on left) were applied topically to the back skin and right ear of mice. After $2 \mathrm{~h}$, unabsorbed particles and DOX solution were removed by wiping. Mouse whole-body fluorescence images were acquired at the indicated time points after application. $\mathbf{b}$ Fluorescence micrographs of frozen sections of the treated area of skin, fixed $48 \mathrm{~h}$ after application, showing brightfield, nuclear counterstain (DAPI) and DOX channel images. Representative images of an experiment repeated 3 times

topically treated subcutaneous B16-F10 melanoma tumors in the skin of mice with 5-FU and/or SPIONs, starting three days post-tumor cell inoculation, then 3 times per week until mice were euthanized. Comparing the growth curves up to day 17 , the latest point at which all mice survived, 5-FU on its own appeared to slightly accelerate 
tumor growth compared with saline (Fig. 3a), but this effect did not reach statistical significance ( $p=0.52$ by repeated measures ANOVA). Topical application of 5 -FU with SPIONs significantly reduced tumor growth compared with topical application of 5 -FU alone $(p=0.0072)$, but SPIONs alone did not $(p=0.96)$. Furthermore, topical co-application of 5-FU with SPIONs significantly improved the survival of mice (i.e., the time for the tumor to reach $1 \mathrm{~cm}$ diameter, the ethical endpoint; Fig. 3b) when compared with 5 -FU alone ( $p=0.012, \log$-rank test).

To determine whether the reduced growth of melanoma tumors in the SPION/5-FU co-administration group was due to an enhanced cytotoxicity, we examined tumors histologically for necrosis. Photomicrographs showed more pronounced necrosis in tumors treated topically with 5-FU + SPIONs than in those treated with 5-FU alone, SPIONs alone or saline vehicle control (Fig. 4a). Quantification by image analysis confirmed that this pro-necrotic effect was statistically significant $(p=0.040$ and $p=0.026$, respectively, by one-way ANOVA with Sidak's multiple comparisons correction) (Fig. 4b). Moreover, combined 5-FU + SPION topical treatment increased the infiltration of tumors by both lymphocytes (Fig. 5a) and macrophages (Fig. 5b), and reduced their vascularization (Fig. 5c) compared with tumors treated topically with 5-FU alone or saline control.
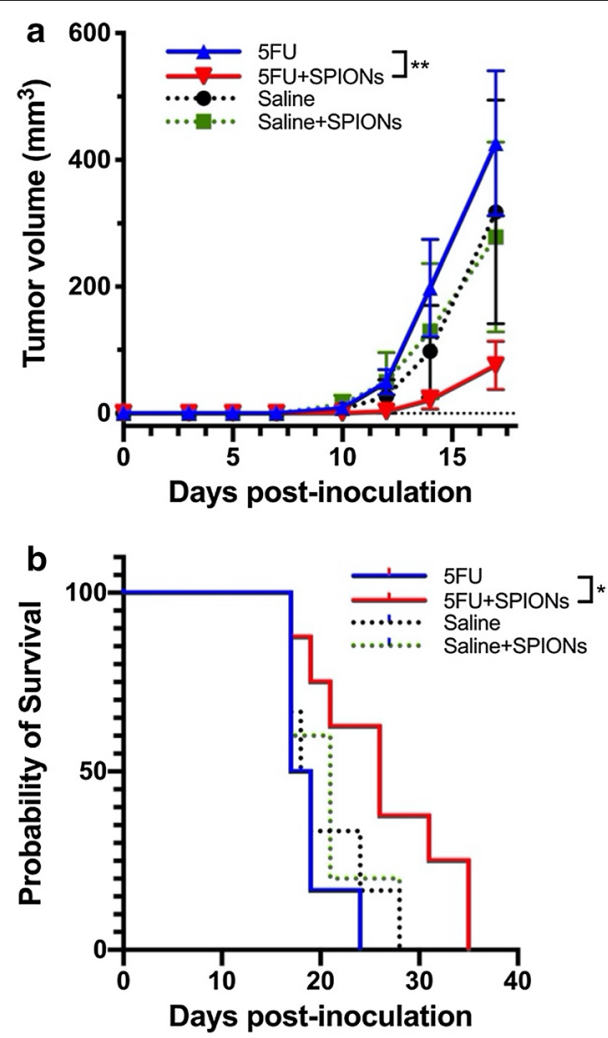

Fig. 3 Topical co-application of 5-FU and SPIONs reduces mouse melanoma tumor growth. Melanoma tumors in 8 week old hairless mice were initiated by subcutaneous injection of $1 \times 10^{4}$ B16F10-tdTomato mouse melanoma cells on the back of the mouse. Three days post cell injection, mice were treated with topical application at the tumor site of $60 \mu \mathrm{L}$ of either saline alone, SPIONs alone, 5-FU (1 mM) alone or 5-FU plus SPIONs ( $1 \mathrm{mg} / \mathrm{mL}$ ) three times a week ( $n=5-8$ mice per group). a Tumor size. ${ }^{* *} p<0.01$, repeated measures ANOVA. $\mathbf{b}$ Survival, in terms of the time taken for the tumor to reach $10 \mathrm{~mm}$ diameter. ${ }^{*} p<0.05$, log-rank (Mantel-Cox) test 


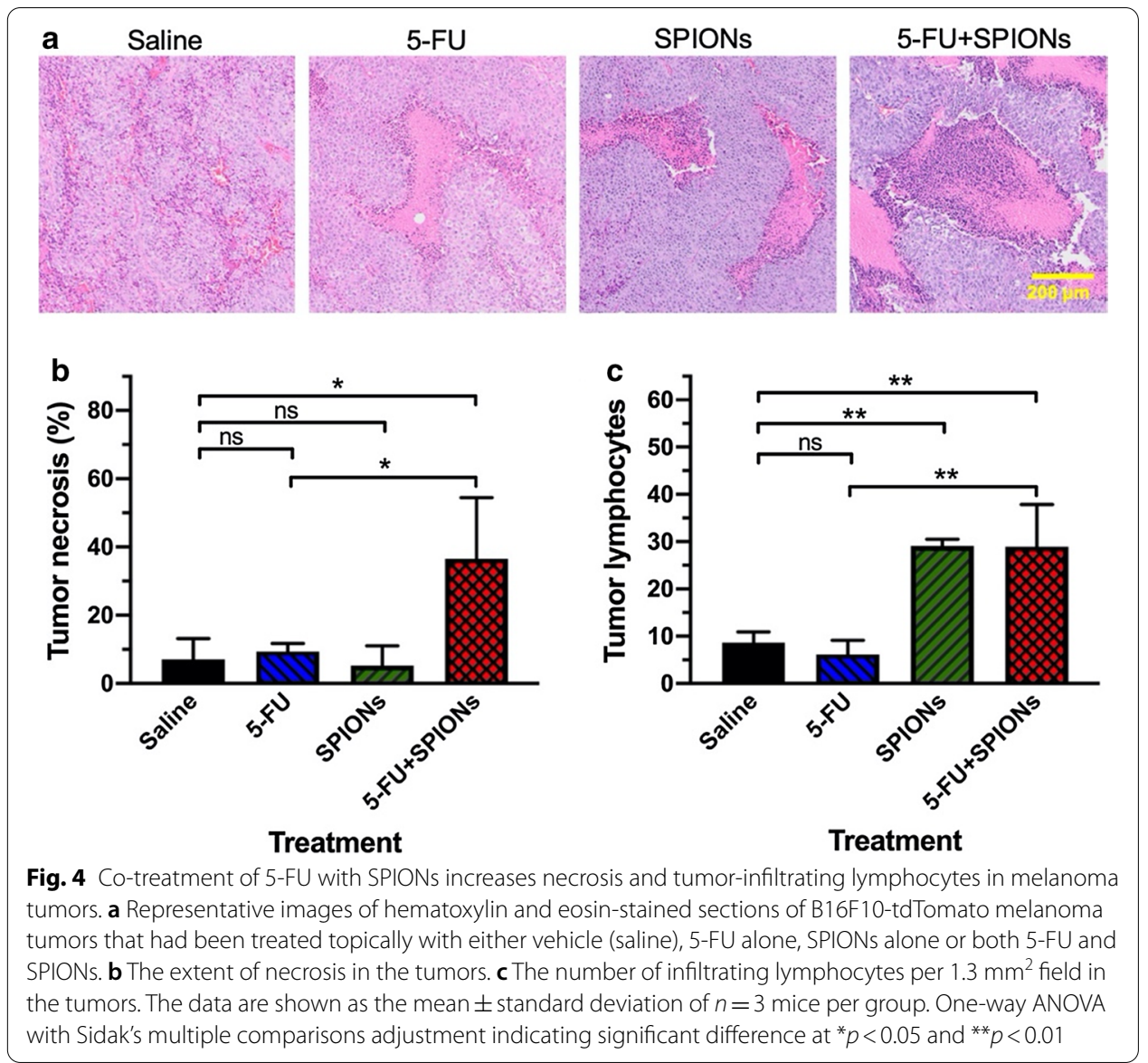

Interestingly, SPIONs alone were able to increase lymphocyte and macrophage infiltration and reduce vascularization (Fig. 5a-c). Thus, similar to the DOX studies, co-administration of SPIONs facilitated the transdermal effects of 5-FU.

\section{Discussion}

There has been a limited number of reports investigating the permeability of inorganic nanoparticles through the skin, with or without conjugated drugs (Baroli et al. 2007; Nose et al. 2012; Anandhakumar and Raichur 2013; Fernandes et al. 2015; Santini et al. 2015; Hsiao et al. 2016; Ozcan et al. 2020). Most previous transdermal nanoparticle studies focused on gold nanoparticles, with studies investigating $\mathrm{Fe}_{3} \mathrm{O}_{4}$ magnetic nanoparticles for topical application rare (Baroli et al. 2007; Rao et al. 2015; Santini et al. 2015). In this study, the excellent penetration through the skin by our SPIONs, despite their relatively large size, might be explained by their steric stabilizers, which prevent the particles from aggregating and accumulating on the skin surface. In addition, $\mathrm{NH}_{2}$ end groups on the coating layer are protonated at low $\mathrm{pH}$, which might facilitate their penetration of the SC.

DOX has been used on its own or in combination with other agents against many cancers, including melanoma, lung, breast and ovarian cancer (Weiss 1992; Pan et al. 2019). However, there are two main concerns of DOX: cardiotoxicity and poor 

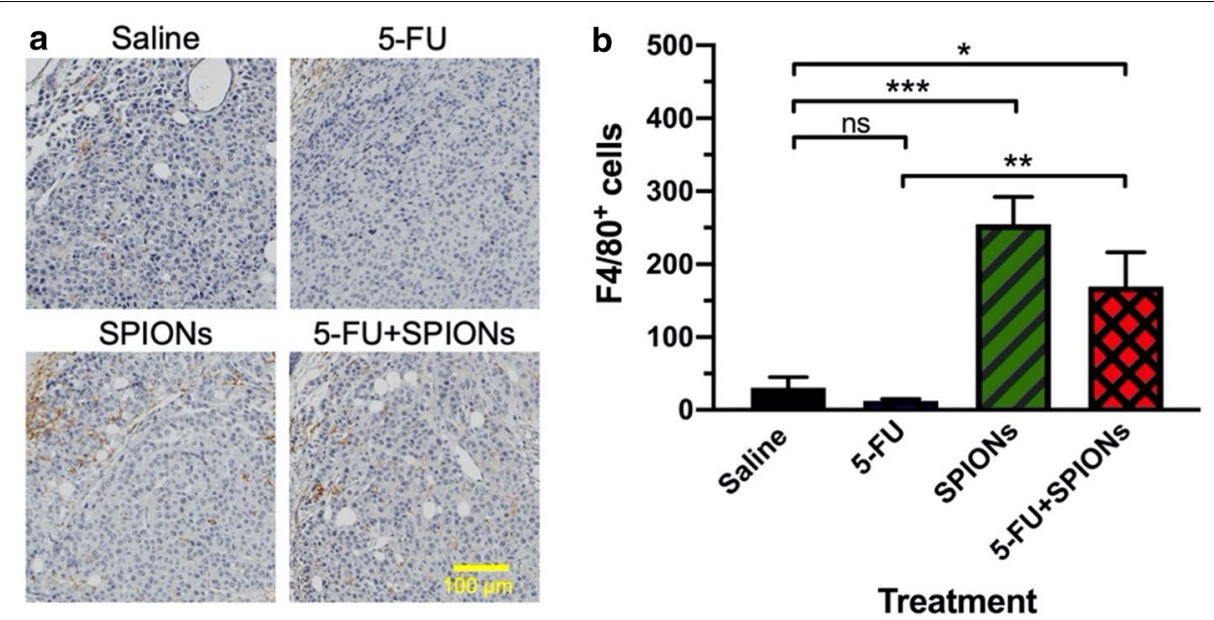
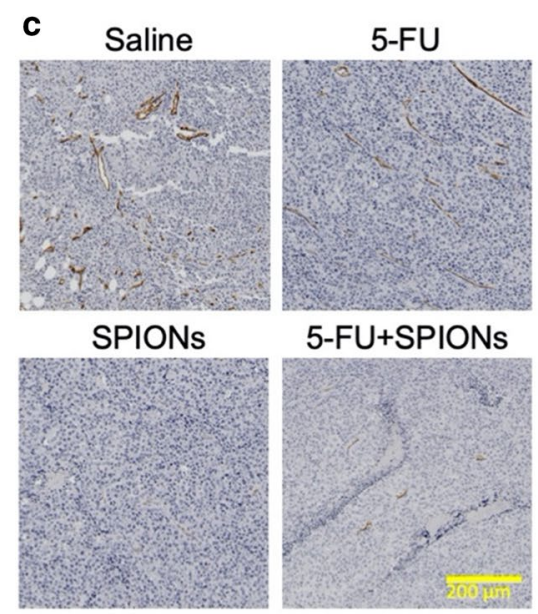

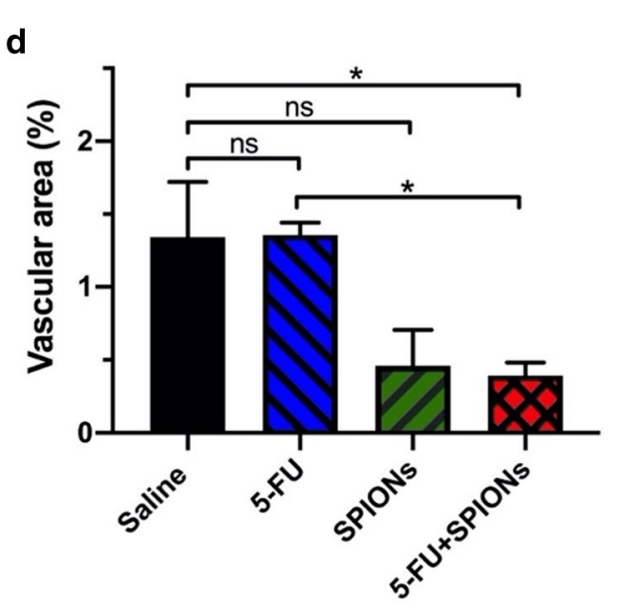

Treatment

Fig. 5 Co-administration of SPIONs with 5-FU increases macrophage infiltration and inhibits blood vessel formation in tumors. a Representative images of B16F10-tdTomato melanoma tumors treated topically with either vehicle (Saline), 5-FU alone, SPIONs alone or both 5-FU and SPIONs. Sections were stained for macrophages with anti-F4/80 antibody (brown). b Quantification of tumor-infiltrating macrophages from F4/80 immunostained sections. c Representative images of melanoma tumors treated topically with vehicle (Saline) 5-FU, SPIONs or both 5-FU and SPIONs. Sections were stained for endothelial cells with anti-CD31 antibody. The data are shown as the mean \pm SEM of $n=5$ measurements from 2 mice per group. d Quantification of vascularization from CD31-immunostained sections. One-way ANOVA followed by Sidak's multiple comparisons adjustment indicating significant difference at ${ }^{*} p<0.05,{ }^{* *} p<0.01$ and ${ }^{* *} p<0.001$

penetration into a solid tumor. To overcome these limitations, the first nano-based, PEGylated liposomal DOX was approved for the treatment of ovarian cancer and Kaposi's sarcoma (Barenholz 2012). Since then, several nanoparticle formulations conjugated or loaded with DOX have been developed for use in cancers (Wang et al. 2011; Chen et al. 2015; Lv et al. 2017; Sheth et al. 2020). However, nanoparticles conjugated or loaded with chemotherapy drugs may be susceptible to inefficient release of drugs at the target site, which is avoided by our adjuvant approach (Bryce et al. 2013). We previously showed that co-administration of $10 \% \mathrm{NH}_{2}$-SPIONs with DOX increased drug penetration into cancer cell spheroids compared with DOX alone (Bryce et al. 2013). The results in this study confirm that co-administration of SPIONs 
additionally enhances the penetration and bioactivity of DOX and 5-FU through the SC and epidermal layers into the dermal layer of the skin of a living animal, without the need for covalently binding DOX to the nanoparticles. The range of molecules whose transdermal penetration is enhanced by these SPIONs without covalent attachment remains to be determined, but conceivably could include other anticancer and immunomodulatory drugs.

For many years, the infiltration of tumors by lymphocytes has been used as a measure of treatment success in clinical trials for melanoma treatment (Rosenberg et al. 1994) and numerous reports indicate that tumor-infiltrating lymphocytes play an essential role in mediating response to chemotherapy in many cancers (Galluzzi et al. 2015). In this study, we showed that SPIONs applied topically to a skin melanoma can enhance the cytotoxicity of 5-FU, increasing necrosis and slowing tumor growth. The increased necrosis may be a combination of enhancing the penetration of 5-FU, increasing leukocyte infiltration and reducing the vascularity of the tumors. The effects of SPIONs on tumor vascularization and immune cell infiltration are independent of 5-FU. A recent study on magnetically localized polyethyleneimine-coated SPIONs also found an enhancement of macrophage infiltration and reduced vascularization of tumors in the absence of cytotoxic drugs (Mulens-Arias et al. 2019), but did not examine the effect of SPIONs on drug delivery.

Chemotherapy of cutaneous melanomas is usually restricted to patients with metastatic disease, in part because of the toxicity of systemically delivered drugs. Further investigation needs to be undertaken to determine the suitability of topical SPION/ drug co-administration for treating premalignant lesions without surgery, as a neoadjuvant treatment for the surgical removal of primary melanomas, which sometimes invade beyond excision margins, and of cutaneous metastases, which can be too numerous to be removed surgically. In particular, the range of molecules whose penetration can be enhanced by the co-administration of specifically stabilized SPIONs needs to be determined.

\section{Conclusion}

Appropriately designed SPIONs show promise for improving the treatment of skinlocalized tumors through a 3-pronged effect: enhancing the immune response by increasing leukocyte infiltration, reducing the nutrient supply by decreasing its vascularity, and enhancing the penetration of chemotherapeutic drugs such as DOX and 5-FU through the skin. The application of such drugs transdermally offers a non-invasive treatment that ensures that the drugs reach their targets while reducing systemic toxicity. We anticipate that these SPIONs could improve the performance of other drugs in current use and enable drugs that had been previously ruled out due to systemic toxicity issues to be revisited.

\section{Supplementary Information}

The online version contains supplementary material available at https://doi.org/10.1186/s12645-021-00079-7. 


\section{Abbreviations}

SC: Stratum corneum; 5-FU: 5-Fluorouracil; DOX: Doxorubicin; SPION: Superparamagnetic iron oxide nanoparticle; RAFT: Reversible addition-fragmentation chain-transfer; MAEP: Monoacryloxyethyl phosphate; MPEG: Poly(ethylene glycol) methyl ether; PEG: Poly(ethylene glycol); DLS: Dynamic light scattering; PBS: Phosphate buffered saline; Rho-SPIONs: Rhodamine-labeled SPIONs; PFA: Paraformaldehyde; DAPI: 4',6-Diamidino-2-phenylindole; H\&E: Hematoxylin and eosin.

\section{Acknowledgements}

The authors acknowledge the instruments and scientific and technical assistance, of Microscopy Australia at the Sydney Microscopy \& Microanalysis, Sydney Cytometry and Sydney Imaging, The University of Sydney; Ms. Stephanie A. Bickley for the synthesis of SPION core; Dr Algi Serelis of DuluxGroup for RAFT reagents.

\section{Authors' contributions}

Conceptualization: VR, BTTP, BSH, JGL; data curation: VR, BTTP, BJK, NTHP; formal analysis: VR, LFK, JGL; funding acquisition: BSH; investigation: VR, BTTP, NTHP, BJK, NP, NCD; methodology: BTTP, BJK, VR, JGL; writing—original draft preparation: VR; writing - review and editing: all authors. All authors read and approved the final manuscript.

\section{Funding}

This study was funded by grants to JGL from the Australasian College of Dermatologists, and to BSH by Sirtex Australia Pty Ltd.

\section{Availability of data and materials}

The datasets used and/or analyzed during the current study are available from the corresponding author on reasonable request.

\section{Ethics approval and consent to participate}

All animal experiments were approved by the University of Sydney's Animal Ethics Committee (AEC 2015/744) and conducted in accordance with the Australian National Health and Medical Research Council guidelines and the Australian Code for the Care and Use of Animals for Scientific Purposes.

\section{Consent for publication}

Not applicable.

\section{Competing interests}

BSH and BTTP are co-authors of patents for the synthesis and use of sterically stabilized nanoparticles: WO 2009/137890 and WO 2012/142669. SKJ holds shares in Zeta Therapeutics Pty Ltd, a company that could potentially benefit from the findings.

\section{Author details}

${ }^{1}$ Key Centre for Polymers and Colloids, School of Chemistry, The University of Sydney, Sydney, NSW, Australia. ${ }^{2}$ Dermatology, Sydney Medical School, and Centenary Institute, The University of Sydney, Sydney, NSW, Australia. ${ }^{3}$ Department of Anatomical Pathology, Conde S. Januário Hospital, Macao, China. ${ }^{4}$ Zeta Therapeutics Pty Ltd, and Sirtex Medical Ltd, Sydney, NSW, Australia. ${ }^{5}$ Present Address: Northern Clinical School and Kolling Institute, The University of Sydney, Sydney, NSW, Australia. ${ }^{6}$ Dermatology, Cancer Services, Royal Prince Alfred Hospital, Sydney, Australia. ${ }^{7}$ Immune Imaging, Centenary Institute, The University of Sydney, Royal Prince Alfred Hospital, Bldg 93, Camperdown, NSW, Australia.

Received: 29 November 2020 Accepted: 27 January 2021

Published online: 06 February 2021

\section{References}

Ali A, Zafar H, Zia M, et al. Synthesis, characterization, applications, and challenges of iron oxide nanoparticles. Nanotechnol Sci App. 2016;9:49-67. https://doi.org/10.2147/NSA.S99986.

Alkilani AZ, McCrudden MTC, Donnelly RF. Transdermal drug delivery: innovative pharmaceutical developments based on disruption of the barrier properties of the stratum corneum. Pharmaceutics. 2015;7:438-70.

Alvarez-Roman R, Merino G, Kalia YN, et al. Skin permeability enhancement by low frequency sonophoresis: lipid extraction and transport pathways. J Pharm Sci. 2003;92:1138-46.

Anandhakumar S, Raichur AM. Polyelectrolyte/silver nanocomposite multilayer films as multifunctional thin film platforms for remote activated protein and drug delivery. Acta Biomater. 2013;9:8864-74.

Barenholz Y. Doxil(R)-the first FDA-approved nano-drug: lessons learned. J Control Release. 2012;160:117-34.

Baroli B, Ennas MG, Loffredo F, et al. Penetration of metallic nanoparticles in human full-thickness skin. J Invest Dermatol. 2007;127:1701-12. https://doi.org/10.1038/sj.jid.5700733.

Bryce NS, Pham BTT, Fong NWS, et al. The composition and end-group functionality of sterically stabilized nanoparticles enhances the effectiveness of co-administered cytotoxins. Biomater Sci. 2013;1:1260-72. https://doi.org/10.1039/ C3BM60120J.

Carter P, Narasimhan B, Wang Q. Biocompatible nanoparticles and vesicular systems in transdermal drug delivery for various skin diseases. Int J Pharm. 2019;555:49-62. https://doi.org/10.1016/j.jpharm.2018.11.032.

Cazares-Delgadillo J, Naik A, Ganem-Rondero A, et al. Transdermal delivery of cytochrome C-A 12.4 kDa protein-across intact skin by constant-current iontophoresis. Pharm Res. 2007;24:1360-8.

Chaudhuri P, Soni S, Sengupta S. Single-walled carbon nanotube-conjugated chemotherapy exhibits increased therapeutic index in melanoma. Nanotechnology. 2010;21:025102.

Chen G, Li D, Li J, et al. Targeted doxorubicin delivery to hepatocarcinoma cells by lactobionic acid-modified laponite nanodisks. New J Chem. 2015;39:2847-55. 
Dianzani C, Zara GP, Maina G, et al. Drug delivery nanoparticles in skin cancers. Biomed Res Int. 2014;2014:895986.

Eamegdool SS, Weible MW, Pham BTT, et al. Ultrasmall superparamagnetic iron oxide nanoparticle prelabelling of human neural precursor cells. Biomaterials. 2014;35:5549-64. https://doi.org/10.1016/j.biomaterials.2014.03.061.

Fang $J-Y$, Hung C-F, Fang Y-P, Chan T-F. Transdermal iontophoresis of 5-fluorouracil combined with electroporation and laser treatment. Int J Pharm. 2004;270:241-9.

Fernandes R, Smyth NR, Muskens OL, et al. Interactions of skin with gold nanoparticles of different surface charge, shape, and functionality. Small. 2015;11:713-21.

Galluzzi L, Buqué A, Kepp O, et al. Immunological effects of conventional chemotherapy and targeted anticancer agents. Cancer Cell. 2015;28:690-714. https://doi.org/10.1016/j.ccell.2015.10.012.

Garbe C, Eigentler TK, Keilholz U, et al. Systematic review of medical treatment in melanoma: current status and future prospects. Oncologist. 2011:16:5-24.

Gautam A, Nanda JS, Samuel JS, et al. Topical delivery of protein and peptide using novel cell penetrating peptide IMTP8. Sci Rep. 2016;6:26278.

Hansen S, Lehr C-M. Nanoparticles for transcutaneous vaccination. Microb Biotechnol. 2012;5:156-67.

Hasany S, Rehman A, Ahmed I, Rajan J. Systematic review of the preparation techniques of iron oxide magnetic nanoparticles. Nanosci Nanotechnol. 2012;2(6):148-58.

Herndon TO, Gonzalez S, Gowrishankar TR, et al. Transdermal microconduits by microscission for drug delivery and sample acquisition. BMC Med. 2004;2:12.

Hsiao PF, Peng S, Tang TC, et al. Enhancing the in vivo transdermal delivery of gold nanoparticles using poly(ethylene glycol) and its oleylamine conjugate. Int J Nanomedicine. 2016;11:1867-78.

Jain N, Wang Y, Jones SK, et al. Optimized steric stabilization of aqueous ferrofluids and magnetic nanoparticles. Langmuir. 2010:26:4465-72.

Kievit FM, Wang FY, Fang C, et al. Doxorubicin loaded iron oxide nanoparticles overcome multidrug resistance in cancer in vitro. J Controlled Release. 2011:152:76-83

Kotiyan P, Majali A, Vavia P, Bhardwaj Y, Sabarwal SA. Electronbeam irradiation: a novel technology for the development of transdermal system of isosorbide dinitrate. Int J Pharm. 2004;270(1):47.

Lohade AA, Jain RR, lyer K, et al. A novel folate-targeted nanoliposomal system of doxorubicin for cancer targeting. AAPS PharmSciTech. 2016;17:1298-311. https://doi.org/10.1208/s12249-015-0462-2.

Lombry C, Dujardin N, Preat V. Transdermal delivery of macromolecules using skin electroporation. Pharm Res. 2000;17:32-7.

Lv Q, He C, Quan F, et al. DOX/LL-2/IFN- $\gamma$ co-loaded thermo-sensitive polypeptide hydrogel for efficient melanoma treatment. Bioact Mater. 2017;3:118-28.

Massart R, Dubois E, Cabuil V, Hasmonay E. Preparation and properties of monodisperse magnetic fluids. J Magn Magn Mater. 1995;149:1-5.

Matsui T, Amagai M. Dissecting the formation, structure and barrier function of the stratum corneum. Int Immunol. 2015;27:269-80. https://doi.org/10.1093/intimm/dxv013.

Mulens-Arias V, Rojas JM, Sanz-Ortega L, et al. Polyethylenimine-coated superparamagnetic iron oxide nanoparticles impair in vitro and in vivo angiogenesis. Nanomedicine Nanotechnol Biol Med. 2019;21:102063. https://doi. org/10.1016/j.nano.2019.102063.

Mundra V, Li W, Mahato RI. Nanoparticle-mediated drug delivery for treating melanoma. Nanomed. 2015;10:2613-33.

Murthy SN, Sammeta SM, Bowers C. Magnetophoresis for enhancing transdermal drug delivery: mechanistic studies and patch design. J Control Release. 2010;148:197-203.

Nose K, Pissuwan D, Goto M, et al. Gold nanorods in an oil-base formulation for transdermal treatment of type 1 diabetes in mice. Nanoscale. 2012:4:3776-80.

Oh JM, Park DH, Choi SJ, Choy JH. LDH nanocontainers as bio-reservoirs and drug delivery carriers. Recent Pat Nanotechnol. 2012;6:200-17.

Ozcan A, Sahin D, Impellizzieri D, et al. Nanoparticle-coupled topical methotrexate can normalize immune responses and induce tissue remodeling in psoriasis. J Invest Dermatol. 2020;140(1003-1014):e8.

Palanisamy S, Wang YM. Superparamagnetic iron oxide nanoparticulate system: synthesis, targeting, drug delivery and therapy in cancer. Dalton Trans. 2019:48:9490-515.

Palmer BC, DeLouise LA. Nanoparticle-enabled transdermal drug delivery systems for enhanced dose control and tissue targeting. Molecules. 2016;21:1719. https://doi.org/10.3390/molecules21121719.

Pan J, Rostamizadeh K, Filipczak N, Torchilin VP. Polymeric co-delivery systems in cancer treatment: an overview on component drugs' dosage ratio effect. Mol Basel Switz. 2019;24:1035.

Pelaz B, Alexiou C, Alvarez-Puebla RA, et al. Diverse applications of nanomedicine. ACS Nano. 2017;11:2313-81. https:// doi.org/10.1021/acsnano.6b06040.

Pham BT, Jain N, Kuchel PW, et al. The interaction of sterically stabilized magnetic nanoparticles with fresh human red blood cells. Int J Nanomedicine. 2015. https://www.dovepress.com/the-interaction-of-sterically-stabilized-magne tic-nanoparticles-with-f-peer-reviewed-article-IJN. Accessed 15 Jun 2020.

Pham TN, Lengkeek NA, Greguric I, et al. Tunable and noncytotoxic PET/SPECT-MRI multimodality imaging probes using colloidally stable ligand-free superparamagnetic iron oxide nanoparticles. Int J Nanomedicine. 2017;12:899-909. https://doi.org/10.2147/IJN.S127171.

Pham BTT, Colvin EK, Pham NTH, et al. Biodistribution and clearance of stable superparamagnetic maghemite iron oxide nanoparticles in mice following intraperitoneal administration. Int J Mol Sci. 2018;19:205.

Prausnitz MR. Microneedles for transdermal drug delivery. Adv Drug Deliv Rev. 2004;56:581-7.

Prausnitz MR, Langer R. Transdermal drug delivery. Nat Biotechnol. 2008:26:1261-8. https://doi.org/10.1038/nbt.1504.

Rao Y, Chen W, Liang X, et al. Epirubicin-loaded superparamagnetic iron-oxide nanoparticles for transdermal delivery: cancer therapy by circumventing the skin barrier. Small. 2015;11:239-47. https://doi.org/10.1002/smll.201400775.

Rosenberg SA, Yannelli JR, Yang JC, et al. Treatment of patients with metastatic melanoma with autologous tumor-infiltrating lymphocytes and interleukin 2. JNCI J Natl Cancer Inst. 1994;86:1159-66. 
Ruifrok A, Johnston D. Quantification of histochemical staining by color deconvolution. Anal Quant Cytol Histol. 2001;23:291-9.

Santini B, Zanoni I, Marzi R, et al. Cream formulation impact on topical administration of engineered colloidal nanoparticles. PLOS ONE. 2015;10:e0126366.

Sheth RA, Wen X, Li J, et al. Doxorubicin-loaded hollow gold nanospheres for dual photothermal ablation and chemoembolization therapy. Cancer Nanotechnol. 2020;11:6. https://doi.org/10.1186/s12645-020-00062-8.

Shubayev VI, Pisanic TR, Jin S. Magnetic nanoparticles for theragnostics. Adv Drug Deliv Rev. 2009;61:467-77.

Tang J-Q, Hou X-Y, Yang C-S, et al. Recent developments in nanomedicine for melanoma treatment. Int J Cancer. 2017;141:646-53. https://doi.org/10.1002/ijc.30708.

Tomayko MM, Reynolds CP. Determination of subcutaneous tumor size in athymic (nude) mice. Cancer Chemother Pharmacol. 1989;24:148-54. https://doi.org/10.1007/BF00300234.

Vanella V, Festino L, Trojaniello C, et al. The role of BRAF-targeted therapy for advanced melanoma in the immunotherapy era. Curr Oncol Rep. 2019;21:76. https://doi.org/10.1007/s11912-019-0827-x.

Vannucci L, Falvo E, Fornara M, et al. Selective targeting of melanoma by PEG-masked protein-based multifunctional nanoparticles. Int J Nanomedicine. 2012;7:1489-509.

Veiseh O, Gunn JW, Zhang M. Design and fabrication of magnetic nanoparticles for targeted drug delivery and imaging. Adv Drug Deliv Rev. 2010;62:284-304.

Wang F, Wang YC, Dou S, et al. Doxorubicin-tethered responsive gold nanoparticles facilitate intracellular drug delivery for overcoming multidrug resistance in cancer cells. ACS Nano. 2011;5:3679-92.

Wang M, Marepally SK, Vemula PK, XU C. Inorganic nanoparticles for transdermal drug delivery and topical application. Nanosci Dermatol. 2016. https://doi.org/10.1016/B978-0-12-802926-8.00005-7.

Weiss RB. The anthracyclines: will we ever find a better doxorubicin? Semin Oncol. 1992;19:670-86.

Yu M, Jambhrunkar S, Thorn P, et al. Hyaluronic acid modified mesoporous silica nanoparticles for targeted drug delivery to CD44-overexpressing cancer cells. Nanoscale. 2013;5:178-83.

Zvyagin AV, Zhao X, Gierden A, et al. Imaging of zinc oxide nanoparticle penetration in human skin in vitro and in vivo. J Biomed Opt. 2008;13:064031.

\section{Publisher's Note}

Springer Nature remains neutral with regard to jurisdictional claims in published maps and institutional affiliations.

- fast, convenient online submission

- thorough peer review by experienced researchers in your field

- rapid publication on acceptance

- support for research data, including large and complex data types

- gold Open Access which fosters wider collaboration and increased citations

- maximum visibility for your research: over 100M website views per year

At BMC, research is always in progress.

Learn more biomedcentral.com/submissions 